\title{
Maximum Flow Finding in Fuzzy Dynamic Graph with the Given Vitality Degree*
}

\author{
Bozhenyuk Alexander \\ Southern Federal University (SFEDU) \\ Rostov-on-Don, Russia \\ e-mail: avb002@yandex.ru
}

\author{
Gerasimenko Evgeniya \\ Southern Federal University (SFEDU) \\ Rostov-on-Don, Russia \\ e-mail: egerasimenko@sfedu.ru
}

\begin{abstract}
The following paper describes the method of the maximum flow finding in fuzzy dynamic graph with the given vitality degree. The peculiarity of the problem is in fuzzy nature of the considered network and given vitality parameters assigned to arcs of the graph. Network's parameters, such as arc capacities and vitality degrees can vary over time, because they have transit character. Such methods can be applied in the real networks solving the task of the optimal cargo transportation.
\end{abstract} flow.

Keywords- fuzzy dynamic graph, fuzzy vitality degree, fuzzy

\section{INTRODUCTION}

The flow tasks arising in the study of transportation networks are relevant due to their wide practical application. Conventional flow tasks allow researchers to find the maximum amount of traffic between selected nodes on the road map, determine the routes of the optimal cost. Dynamic networks describe complex systems, which parameters can vary over time. Algorithms underlying these tasks allow solving optimization problems taking into account the flow departure time, transit times along the arcs of the graph.

However, the flow tasks and methods of its solution are presented in the references in crisp conditions, while changes in environment, variations in petrol prices, measurement errors influence such network parameters, as arc capacities and transmission costs. Therefore, these tasks should be considered in the fuzzy conditions and we turn to the fuzzy graphs for solving such a problems.

Dynamic tasks considered in the conventional flow literature take into account transit times along the arcs of the graph, while network's parameters are constant. We suggest to consider dependence of arc capacities and transmission costs on flow departure time and operate with fully dynamic networks instead of stationary-dynamic ones [1], using the notions of the time-expanded graphs.

Vitality parameter peculiar to arcs of the network usually isn't taken into account while studying networks. Nowadays, the vitality of the network is studied less, while railways and roads include the complex objects, such as stations, distillation ways, culverts, wagon, passenger and cargo managements. Thus, conventional definition of "vitality" was introduced by the authors H. Frank and I. Frisch in [2] as sensitivity of the network to damages. However, vitality applied to the networks

*This work has been supported by the Russian Foundation for Basic Research, Project № 16-01-00090 a, and the Ministry of Education and Science of the Russian Federation under Project № 213.01-11/2014-48 (Base part, State task 2014/174) is ability of its objects and links among them to be resistant to weather conditions, traffic accidents and its combinations, and save and restore (fully or partially) objects themselves and their connections, arc capacities of the network's sections in case of damage. Sometimes network's parameters can be set qualitatively. Thus, one can set the notion "vitality degree" considering the roads and railways. In this case "vitality degree" is considered as probability of trouble-free operation of the road section and some subjective value, such as importance and reliability, etc.

Therefore, the method of the maximum flow finding in fuzzy dynamic graph with the given vitality degree is proposed in the present paper. The paper is structured as follows. In the Section 2 we give literature review of the flow and vitality problems in networks. Section 3 contains basic concepts and definitions. Section 4 presents the proposed method of the maximum flow finding in fuzzy dynamic graph with the given vitality degree. Section 5 provides numerical example illustrating the main steps of the proposed method.

\section{LITERATURE REVIEW}

Historically, the task of the maximum flow determining was the first flow task in dynamic graphs, described in the literature. Authors L. Ford and D. Fulkerson [3] introduce the notion "dynamic flow".

Initially there were two methods for solving such a problem. The first method assumes using of the "timeexpanded graph" $[3,4]$, that includes constructing the nodes and arcs copies in the given time dimension. The second implements the minimum cost flow method, in particular, the shortest path algorithm considering the flow transit time along the arc equals the transmission cost along the corresponding arc.

Authors E. Minieka and J. Aronson [5, 6] determine dynamic networks either. In particular, E. Minieka [5] simulated a situation, when some arcs of the dynamic graph can't be available at some time periods. Author Orlin [7] presents the solution of the maximum dynamic flow problem in the case of either discrete or continuous time periods.

Dynamic nature of the network's parameters and their opportunity to change in time isn't taken into account in conventional sources that is incorrect in general. The authors 
suggest calling these networks "stationary-dynamic" and introducing the notion of dynamic networks in [1].

However, the maximum dynamic flow problem was hardly considered in the literature in fuzzy conditions. The factors lead to the flow problem statements in fuzzy conditions are considered in [8].The authors of the present paper proposed formal methods for solving this problem in fuzzy conditions and applied method that operated the central values of fuzzy numbers and blurred them in the terminal step of the algorithm [9]. At the same time, proposed method doesn't consider the vitality, peculiar to the network's parameters, so the necessity to develop such a method will appear.

H. Frank and I. Frisch were the first authors, who examined vitality in terms of the networks. In $[10,11]$ searching and increasing of the network's vitality and finding the centers with the largest vitality degree are considered. However, vitality finding wasn't considered in the conventional literature in terms of flows in networks. Though, it is important parameter, which reflects reliability and robustness of the network's parameters and in many cases it is important to set this parameter to the arc of graph. Accordingly, maximum flow finding algorithm in the fuzzy dynamic graph with given vitality degree was proposed.

\section{BASIC CONCEPTS AND DEFINITIONS}

Let us present the basic concepts, definitions and rules underlying the proposed method. The concepts of the fuzzy stationary-dynamic network and fuzzy dynamic network are presented in the following definitions $\mathbf{1}$ and $\mathbf{2}$.

\section{A. Definition 1}

Fuzzy stationary-dynamic network [1] is a network in the form of the fuzzy directed graph $\tilde{G}=(X, \tilde{A})$, where $X=\left\{x_{1}, x_{2}, \ldots, x_{n}\right\} \quad-\quad$ the set of nodes, $\tilde{A}=\left\{<\mu_{\tilde{A}}<x_{i}, x_{j}>/<x_{i}, x_{j}>\right\},<x_{i}, x_{j}>\in X^{2}, \mu_{\tilde{A}}<x_{i}, x_{j}>$ - fuzzy set of arcs, where $\mu_{\tilde{A}}\left(x_{i}, x_{j}\right)$ is the membership degree of the directed arc $\left\langle x_{i}, x_{j}\right\rangle$ to the fuzzy set of directed arcs $\tilde{A}$. Fuzzy arc capacity $\tilde{u}_{i j}$ is used as $\mu_{\tilde{A}}\left(x_{i}, x_{j}\right)$. Transit time $\tau_{i j}$ is set to each arc of the graph. There is time horizon $T=\{0,1, \ldots, p\}$, which determines, that all flow units sent from the source must arrive at the sink not later, than in the time $p$. $\tau_{i j}$ is a positive number.

\section{B. Definition 2}

Fuzzy dynamic network [1] is a network $\tilde{G}=(X, \tilde{A})$, where $X=\left\{x_{1}, x_{2}, \ldots, x_{n}\right\} \quad-\quad$ the set of nodes, $\tilde{A}=\left\{<\mu_{\tilde{A}}<x_{i}, x_{j}>/<x_{i}, x_{j}>\right\},<x_{i}, x_{j}>\in X^{2}, \mu_{\tilde{A}}<x_{i}, x_{j}>$ - fuzzy set of arcs, where $\mu_{\tilde{A}}\left(x_{i}, x_{j}\right)$ is the membership degree of the directed arc $\left\langle x_{i}, x_{j}\right\rangle$ to the fuzzy set of directed arcs $\tilde{A}$. Transit arc capacity $\tilde{u}_{i j}(\theta)$ and transit time $\tau_{i j}(\theta)$, where $\theta \in T=\{0,1, \ldots, p\}-$ the flow departure time, are set to each arc of the graph. There is time horizon $T=\{0,1, \ldots, p\}$, which determines, that all flow units sent from the source must arrive at the sink not later, than in the time $p . \tau_{i j}$ is a positive number.

The main concept of the proposed method is the concept of the time-expanded graph that constructed from the initial dynamic graph according to the definition 3.

\section{Definition 3}

Time-expanded fuzzy static graph [3], [12] $\tilde{G}_{p}$ is the graph, constructed from the given fuzzy dynamic graph $\tilde{G}$ by expanding the original dynamic graph in the time dimension by making a separate copy of every node $x_{i} \in X$ at every time $\theta \in T$. This copy is called "node-time pair" $\left(x_{i}, \theta\right)$.

Fuzzy directed way $\tilde{P}\left(x_{i}, x_{m}\right)$ of the graph $\tilde{G}=(X, \tilde{A})$ is a sequence of fuzzy directed arcs from the node $x_{i}$ to the node $x_{m}:$

$$
\begin{aligned}
& \tilde{P}\left(x_{i}, x_{m}\right)=<\mu_{\tilde{A}}<x_{i}, x_{j}>/<x_{i}, x_{j}>>, \\
& \mu_{\tilde{A}}<x_{j}, x_{k}>/<x_{j}, x_{k}>>, \ldots, \mu_{\tilde{A}}<x_{l}, x_{m}>/<x_{l}, x_{m}>>.
\end{aligned}
$$

Conjunctive durability of the way $\mu\left(\tilde{P}\left(x_{i}, x_{m}\right)\right)$ is defined as

$$
\mu\left(\tilde{P}\left(x_{i}, x_{m}\right)\right)=\underset{<x_{\alpha}, x_{\beta}>\in \tilde{P}\left(x_{i}, x_{m}\right)}{\&} \mu_{\tilde{A}}<x_{\alpha}, x_{\beta}>.
$$

Fuzzy directed way $\tilde{P}\left(x_{i}, x_{m}\right)$ is called a simple way between vertices $x_{i}$ and $x_{m}$ if its part is not a way between the same vertices.

Vertex $y$ is called a fuzzy accessible from the vertex $x$ in the graph $\tilde{G}=(X, \tilde{A})$ if the fuzzy directed way from the node $x$ to the node $y$ exists.

The accessible degree of the node $y$ from the node $x,(x \neq y)$ is defined by the following expression:

$$
\gamma(x, y)=\max _{\alpha}\left(\mu\left(\tilde{P}_{\alpha}(x, y)\right), \alpha=1,2, \ldots, p,\right.
$$

where $p$ is the number of various simple directed ways from vertex $x$ to vertex $y$.

We consider the degree of fuzzy graph vitality as a degree of strong connection [10,11], so it will be defined by the following expression:

$$
V(\tilde{G})=\&_{x_{i} \in X} \underset{x_{j} \in X}{\&} \gamma\left(\mathrm{x}_{\mathrm{i}}, \mathrm{x}_{\mathrm{j}}\right) .
$$

It means that there is a route between each pair of the graph vertices with a conjunctive strength not less than value $V$.

Let us present the rule of the time-expanded graph construction in terms of the maximum dynamic flow finding with the given vitality degree. 


\section{Rule 1}

Let $\tilde{G}_{p}=\left(X_{p}, \tilde{A}_{p}\right)$ represent fuzzy time-expanded static graph of the original dynamic fuzzy graph. The set of nodes $X_{p}$ of the graph $\tilde{G}_{p}$ is defined as $X_{p}=\left\{\left(x_{i}, \theta\right):\left(x_{i}, \theta\right) \in X \times T\right\}$. The set of arcs $\tilde{A}_{p}$ consists of arcs from each node-time pair $\left(x_{i}, \theta\right) \in X_{p}$ to every node-time pair $\quad\left(x_{j}, \vartheta=\theta+\tau_{i j}(\theta)\right), \quad$ where $\quad x_{j} \in \Gamma\left(x_{i}\right) \quad$ and $\theta+\tau_{i j}(\theta) \leq p$. Fuzzy arc capacities $\tilde{u}\left(x_{i}, x_{j}, \theta, \vartheta\right)$ joining $\left(x_{i}, \theta\right)$ with $\left(x_{j}, \vartheta\right)$ are equal to $\tilde{u}_{i j}(\theta)$. Transit times $\tau\left(x_{i}, x_{j}, \theta, \vartheta\right)$ joining $\left(x_{i}, \theta\right)$ with $\left(x_{j}, \vartheta\right)$ are equal to $\tau_{i j}(\theta)$. Vitality parameters $v\left(x_{i}, x_{j}, \theta, \vartheta\right)$ connecting $\left(x_{i}, \theta\right)$ with $\left(x_{j}, \vartheta\right)$ are equal to $v_{i j}(\theta)$.

\section{PROPOSED METHOD}

Consider the problem of the maximum flow finding in fuzzy dynamic graph with the given vitality degree as a model (1)-(6).

\section{Maximize $\tilde{v}(p)$}

$$
\sum_{\theta=0}^{p}\left(\sum_{x_{j} \in \Gamma\left(x_{i}\right)} \tilde{\xi}_{i j}(\theta)-\sum_{x_{j} \in \Gamma^{-1}\left(x_{i}\right)} \tilde{\xi}_{j i}\left(\theta-\tau_{j i}(\theta)\right)\right)=\tilde{v}(p), x_{i}=s
$$

$$
\begin{gathered}
\sum_{\theta=0}^{p}\left(\sum_{x_{j} \in \Gamma\left(x_{i}\right)} \tilde{\xi}_{i j}(\theta)-\sum_{x_{j} \in \Gamma^{-1}\left(x_{i}\right)} \tilde{\xi}_{j i}\left(\theta-\tau_{j i}(\theta)\right)=\tilde{0}, x_{i} \neq s, t ; \theta \in T\right. \\
\sum_{\theta=0}^{p}\left(\sum_{x_{j} \in \Gamma\left(x_{i}\right)} \tilde{\xi}_{i j}(\theta)-\sum_{x_{j} \in \Gamma^{-1}\left(x_{i}\right)} \tilde{\xi}_{j i}\left(\theta-\tau_{j i}(\theta)\right)\right)=-\tilde{v}(p), x_{i}=t \\
\tilde{0} \leq \tilde{\xi}_{i j}(\theta) \leq \tilde{u}_{i j}(\theta), \forall\left(x_{i}, x_{j}\right) \in \tilde{A}, \theta \in T \\
v_{i j}(\theta) \geq v_{r e q}, \forall\left(x_{i}, x_{j}\right) \in \tilde{A}, \theta \in T
\end{gathered}
$$

Equation (1) means that we maximize fuzzy amount of flow $\tilde{v}$ for $p$ periods of time, i.e. $\tilde{v}(p)$. The equation (2) indicates that the maximum flow value $\tilde{v}$ for $p$ time periods is equal to the flow, leaving the source for $p$ time periods $\sum_{\theta=0}^{p} \sum_{x_{j} \in \Gamma\left(x_{i}\right)} \tilde{\xi}_{i j}(\theta), x_{i}=s$. The equation (4) reflects that the maximum flow value $\tilde{v}$ for $p$ time periods is equal to the total flow entering the sink for $p$ time periods $\sum_{\theta=0}^{p} \sum_{x_{j} \in \Gamma^{-1}\left(x_{i}\right)} \tilde{\xi}_{j i}\left(\theta-\tau_{j i}(\theta)\right), x_{i}=t$. The total amount of flow entering the source $\sum_{\theta=0}^{p} \sum_{x_{j} \in \Gamma^{-1}\left(x_{i}\right)} \tilde{\xi}_{j i}\left(\theta-\tau_{j i}(\theta)\right), x_{i}=s$ for $p$ time periods is equal to the total flow $\sum_{\theta=0}^{p} \sum_{x_{j} \in \Gamma\left(x_{i}\right)} \tilde{\xi}_{i j}(\theta), x_{i}=t$ leaving the sink for $p$ time periods and is equal to $\tilde{0}$. For each node $x_{i}$ except the source and the sink and for each time period $\theta$ the amount of flow $\sum_{\theta=0}^{p} \sum_{x_{j} \in \Gamma^{-1}\left(x_{i}\right)} \tilde{\xi}_{j i}\left(\theta-\tau_{j i}(\theta)\right), x_{i} \neq s, t$ entering $x_{i}$ at each period of time $\left(\theta-\tau_{j i}(\theta)\right)$ is equal to the total amount of flow $\sum_{\theta=0}^{p} \sum_{x_{j} \in \Gamma\left(x_{i}\right)} \tilde{\xi}_{i j}(\theta), x_{i} \neq s, t$ leaving $x_{i}$ at time $\theta$ for $p$ time periods as stated in (3). The inequality (5) indicates that the flows $\tilde{\xi}_{i j}(\theta)$ for all time periods should be less than arc capacities of the corresponding arcs $\tilde{u}_{i j}(\theta)$ in the same time periods. The inequality (6) reflects that the vitality degree $v_{i j}(\theta)$ for all arcs for all time periods should not be less than required vitality degree $v_{r e q}$.

Step 1. Go to the time-expanded fuzzy static graph $\tilde{G}_{p}$ from the given fuzzy dynamic graph $\tilde{G}$ by expanding the original dynamic graph in the time dimension by making a separate copy of every node $x_{i} \in X$ at each time $\theta \in T$ according to the rule 1 . It is necessary to calculate the maximum dynamic flow leaving the group of sources, expanded for $p$ periods and entering the group of sinks expanded for $p$ periods no later than $p$. Let us introduce an artificial source $s^{\prime}$ and sink $t^{\prime}$ and join $s^{\prime}$ and $t^{\prime}$ by arcs with each true source and sink, respectively. Artificial arcs leaving and entering artificial nodes have infinite capacities and vitality degree equals 1 .

Step 2. Build a fuzzy residual network $\tilde{G}_{p}^{\mu}=\left(X_{p}^{\mu}, \tilde{A}_{p}^{\mu}\right)$ depending on the flow values going along the arcs of the graph $\tilde{G}_{p}$. If the following condition is satisfied:

$$
\left\{\begin{array}{l}
\tilde{\xi}\left(x_{i}, x_{j}, \theta, \vartheta\right)<\tilde{u}\left(x_{i}, x_{j}, \theta, \vartheta\right), \\
v\left(x_{i}, x_{j}, \theta, \vartheta\right) \geq v_{r e q}
\end{array}\right.
$$

then $\tilde{u}^{\mu}\left(x_{i}, x_{j}, \theta, \vartheta\right)=\tilde{u}\left(x_{i}, x_{j}, \theta, \vartheta\right)-\tilde{\xi}\left(x_{i}, x_{j}, \theta, \vartheta\right)$.

If the following condition is satisfied:

$$
\left\{\begin{array}{l}
\tilde{\xi}\left(x_{i}, x_{j}, \vartheta, \theta\right)>\tilde{0}, \\
v\left(x_{i}, x_{j}, \theta, \vartheta\right) \geq v_{r e q} .
\end{array}\right.
$$

Then $\tilde{u}^{\mu}\left(x_{j}, x_{i}, \vartheta, \theta\right)=\tilde{\xi}\left(x_{i}, x_{j}, \theta, \vartheta\right)$. 
Initially, residual network coincides with the "timeexpanded" static graph $\left(\right.$ as $\left.\left.\tilde{\xi}\left(x_{i}, x_{j}, \theta, \vartheta\right)=\tilde{0}\right)\right)$.

Step 3. Find the augmenting shortest path $\tilde{P}_{p}^{\mu}$ in terms of the number of arcs from the artificial source $s$ to the artificial sink $t^{\prime}$ in the constructed fuzzy residual network $\tilde{G}_{p}^{\mu}$. The choice of the shortest path is according to breadth-first search.

3.1. Go to the step 4 if the augmenting path $\tilde{P}_{p}^{\mu}$ is found.

3.2 Fuzzy maximum flow value is $\tilde{\xi}\left(x_{i}, x_{j}, \theta, \vartheta\right)+\tilde{\delta}_{p}^{\mu} \times \tilde{P}_{p}^{\mu}=\tilde{v}(p)$ in the time-expanded fuzzy static graph if the path is failed to find from $s^{\prime}$ to $t^{\prime}$, then go to the step 6.

Step 4. Pass the maximum amount of flow units depending on the arc with minimal fuzzy residual capacity $\tilde{\delta}_{p}^{\mu}=\min \left[\tilde{u}\left(\tilde{P}_{p}^{\mu}\right)\right], \quad \tilde{u}\left(\tilde{P}_{p}^{\mu}\right)=\min \left[\tilde{u}^{\mu}\left(x_{i}, x_{j}, \theta, \vartheta\right)\right]$, $\left(x_{i}, \theta\right),\left(x_{j}, \vartheta\right) \in \tilde{P}_{p}^{\mu}$ along the found path

Step 5. Update the fuzzy flow values in the graph $\tilde{G}_{p}$ : replace the fuzzy flow $\tilde{\xi}\left(x_{j}, x_{i}, \theta, \vartheta\right)$ by $\tilde{\xi}\left(x_{j}, x_{i}, \theta, \vartheta\right)-\tilde{\delta}_{p}^{\mu}$ for arcs connecting node-time pair $\left(x_{i}^{\mu}, \vartheta\right)$ with $\left(x_{j}^{\mu}, \theta\right)$, such as $\left(\left(x_{i}^{\mu}, \vartheta\right),\left(x_{j}^{\mu}, \theta\right)\right) \notin \tilde{A}_{p},\left(\left(x_{i}^{\mu}, \vartheta\right),\left(x_{j}^{\mu}, \theta\right)\right) \in \tilde{A}_{p}^{\mu}$ in $\tilde{G}_{p}^{\mu}$ along the corresponding arcs going from $\left(x_{j}, \theta\right)$ to $\left(x_{i}, \vartheta\right)$ from $\tilde{G}_{p}$ and replace the fuzzy flow $\tilde{\xi}\left(x_{i}, x_{j}, \theta, \vartheta\right)$ by $\tilde{\xi}\left(x_{i}, x_{j}, \theta, \vartheta\right)+\tilde{\delta}_{p}^{\mu}$ for arcs connecting node-time pair $\left(x_{i}^{\mu}, \theta\right)$ with $\left(x_{j}^{\mu}, \vartheta\right)$, such as $\left(\left(x_{i}^{\mu}, \vartheta\right),\left(x_{j}^{\mu}, \theta\right)\right) \in \tilde{A}_{p}^{\mu}$ in $\tilde{G}_{p}^{\mu}$ along the arcs going from $\left(x_{i}, \theta\right)$ to $\left(x_{j}, \vartheta\right)$ from $\tilde{G}_{p}$. Replace the fuzzy flow value in $\tilde{G}_{p}$ : $\tilde{\xi}\left(x_{i}, x_{j}, \theta, \vartheta\right) \rightarrow \tilde{\xi}\left(x_{i}, x_{j}, \theta, \vartheta\right)+\tilde{\delta}_{p}^{\mu} \times \tilde{P}_{p}^{\mu}$ and go to the step 2, starting with the updated flow values.

Step 6. Turn to the original dynamic fuzzy graph $\tilde{G}$ if the maximum flow $\tilde{\xi}\left(x_{i}, x_{j}, \theta, \vartheta\right)+\tilde{\delta}_{p}^{\mu} \times \tilde{P}_{p}^{\mu}=\tilde{v}(p)$ from the artificial source $s^{\prime}$ to the artificial sink $t^{\prime}$ defined by the set of paths $\tilde{P}_{p}^{\mu}$ is found in $\tilde{G}_{p}$ such a way: cancel the artificial arcs and artificial nodes $s^{\prime}$ and $t^{\prime}$. Therefore, the maximum flow of the value $\tilde{v}(p)$ is obtained in the original graph, which is equivalent to the flow from the sources (initial node, expanded on the $p$ periods) to the sinks (terminal node, expanded on the $p$ periods) in $\tilde{G}_{p}$ after cancelling artificial nodes.

\section{A. Calculations with fuzzy numbers}

Note, that fuzzy arc capacities can be represented in any form of fuzzy numbers. Let in our case fuzzy arc capacities are set as fuzzy trapezoidal numbers.

Since the calculations with trapezoidal numbers are cumbersome and result in strong blurring of the resulting number's borders, we suggest to operate fuzzy numbers according to the method, described in [9]. In this case we will operate the central values of fuzzy numbers in the form of the fuzzy intervals, blurring the result at the final step and presenting it as a trapezoidal the number.

Let us introduce the following equation for the left $l^{L}$ and the right $l^{R}$ trapezoidal deviation borders, as in (7)-(8):

$$
\begin{aligned}
& l^{L}=\frac{\left(a_{2}-a^{\prime}\right)}{\left(a_{2}-a_{1}\right)} \times l_{1}^{L}+\left(1-\frac{\left(a_{2}-a^{\prime}\right)}{\left(a_{2}-a_{1}\right)}\right) \times l_{2}^{L}, \\
& l^{R}=\frac{\left(b_{2}-b^{\prime}\right)}{\left(b_{2}-b_{1}\right)} \times l_{1}^{R}+\left(1-\frac{\left(b_{2}-b^{\prime}\right)}{\left(b_{2}-b_{1}\right)}\right) \times l_{2}^{R} .
\end{aligned}
$$

In (7)-(8) $l^{L}$ - the left deviation border of the fuzzy trapezoidal number with the center $\left[a^{\prime}, b^{\prime}\right] ; l^{R}-$ the right deviation border of the fuzzy trapezoidal number with the center $\left[a^{\prime}, b^{\prime}\right]$. It is represented in the Fig. 1.

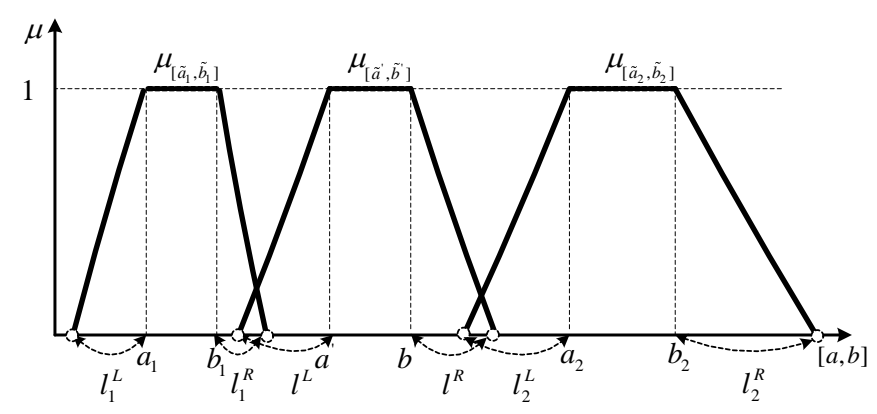

Fig. 1. Defining of the membership function $\mu_{\tilde{a}^{\prime}}(a)$

During the algorithm we should produce operations of adding and subtraction of fuzzy intervals.

Let two fuzzy intervals $\tilde{A}_{1}=\left[a_{1}, b_{1}\right]$ и $\tilde{A}_{2}=\left[a_{2}, b_{2}\right]$ are given .

We carry out calculations according to the equation of nonstandard subtraction of fuzzy intervals, as in (9).

$$
\tilde{A}_{1}-\tilde{A}_{2}=\left[\min \left\{a_{1}-a_{2}, b_{1}-b_{2}\right\}, \max \left\{a_{1}-a_{2}, b_{1}-b_{2}\right\}\right] .
$$

This formula, unlike the classic one, does not lead to the strong blurring of the resulting borders and does not produce negative flow values, that is unacceptable for flows.

\section{NUMERICAL EXAMPLE}

Let us represent the proposed method based on the numerical example. Let the initial fuzzy dynamic graph is represented in the Fig. 2 It is necessary to find the maximum 
flow in the initial dynamic graph with the vitality degree no less than 0,6 and represent the result in the form of the fuzzy trapezoidal number.

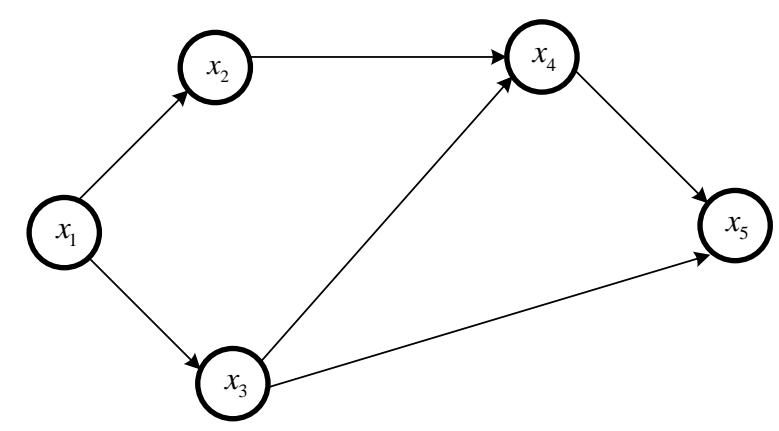

Fig. 2. Initial fuzzy dynamic graph $\tilde{G}$

Fuzzy arc capacities depend on the flow departure time are presented in the Table I.

TABLE I. FUZZY ARC CAPACITIES $\tilde{u}_{i j}$, DEPEND ON FLOW DEPARTURE THE TIME $\theta$

\begin{tabular}{|c|c|c|c|c|}
\hline \multirow{2}{*}{$\begin{array}{l}\text { Arcs of the } \\
\text { graph }\end{array}$} & \multicolumn{4}{|c|}{ Fuzzy arc capacities $\tilde{u}_{i j}$ at the moment $\theta$, units. } \\
\hline & 0 & 1 & 2 & 3 \\
\hline$\left(x_{1}, x_{2}\right)$ & {$[3 \tilde{0}, 4 \tilde{2}]$} & {$[\tilde{8}, 1 \tilde{0}]$} & {$[5 \tilde{0}, 7 \tilde{1}]$} & {$[\tilde{8}, 1 \tilde{0}]$} \\
\hline$\left(x_{1}, x_{3}\right)$ & {$[5 \tilde{0}, 7 \tilde{1}]$} & {$[2 \tilde{0}, 2 \tilde{9}]$} & {$[2 \tilde{0}, 2 \tilde{9}]$} & {$[10 \tilde{0}, 13 \tilde{2}]$} \\
\hline$\left(x_{2}, x_{4}\right)$ & {$[1 \tilde{2}, 1 \tilde{6}]$} & {$[2 \tilde{1}, 3 \tilde{0}]$} & {$[3 \tilde{0}, 4 \tilde{2}]$} & {$[5 \tilde{5}, 7 \tilde{7}]$} \\
\hline$\left(x_{3}, x_{4}\right)$ & {$[3 \tilde{7}, 5 \tilde{0}]$} & {$[7 \tilde{0}, 9 \tilde{5}]$} & {$[6 \tilde{0}, 8 \tilde{3}]$} & {$[6 \tilde{0}, 8 \tilde{3}]$} \\
\hline$\left(x_{3}, x_{5}\right)$ & {$[1 \tilde{2}, 1 \tilde{6}]$} & {$[1 \tilde{5}, 2 \tilde{1}]$} & {$[1 \tilde{5}, 2 \tilde{1}]$} & {$[1 \tilde{9}, 2 \tilde{7}]$} \\
\hline$\left(x_{4}, x_{5}\right)$ & {$[1 \tilde{1}, 1 \tilde{5}]$} & {$[3 \tilde{5}, 4 \tilde{8}]$} & {$[4 \tilde{4}, 5 \tilde{9}]$} & {$[10 \tilde{9}, 13 \tilde{4}]$} \\
\hline
\end{tabular}

Table II gives the transit times along the arcs depend on the flow departure time.

TABLE II. TRANSIT TIMES ALONG THE ARCS, DEPEND ON THE FLOW DEPARTURE TIME $\theta$

\begin{tabular}{|c|l|l|l|l|}
\hline \multirow{2}{*}{ Arcs of the graph } & \multicolumn{4}{|c|}{ Transit times $\tau_{i j}$ at time periods $\theta$, time units. } \\
\cline { 2 - 5 } & \multicolumn{1}{|c|}{$\boldsymbol{1}$} & \multicolumn{1}{|c|}{$\mathbf{2}$} & $\mathbf{3}$ \\
\hline$\left(x_{1}, x_{2}\right)$ & 1 & 1 & 1 & 2 \\
\hline$\left(x_{1}, x_{3}\right)$ & 1 & 1 & 3 & 2 \\
\hline$\left(x_{2}, x_{4}\right)$ & 5 & 1 & 3 & 1 \\
\hline$\left(x_{3}, x_{4}\right)$ & 1 & 1 & 2 & 2 \\
\hline$\left(x_{3}, x_{5}\right)$ & 4 & 2 & 1 & 2 \\
\hline$\left(x_{4}, x_{5}\right)$ & 5 & 1 & 1 & 3 \\
\hline
\end{tabular}

Vitality degrees that depend on the flow departure time are set in the Table III.

TABLE III. VITALITY PARAMETERS, DEPEND ON THE FLOW DEPARTURE TIME $\theta$

\begin{tabular}{|c|c|c|c|c|}
\hline \multirow[t]{2}{*}{$\begin{array}{l}\text { Arcs of the } \\
\text { graph }\end{array}$} & \multicolumn{4}{|c|}{ 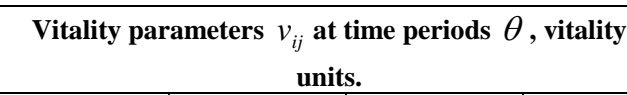 } \\
\hline & 0 & 1 & 2 & 3 \\
\hline$\left(x_{1}, x_{2}\right)$ & 0,8 & 0,9 & 0,3 & 0,5 \\
\hline$\left(x_{1}, x_{3}\right)$ & 0,6 & 0,7 & 0,4 & 0,8 \\
\hline$\left(x_{2}, x_{4}\right)$ & 0,5 & 0,6 & 0,3 & 0,3 \\
\hline$\left(x_{3}, x_{4}\right)$ & 0,3 & 0,9 & 0,9 & 0,8 \\
\hline$\left(x_{3}, x_{5}\right)$ & 0,7 & 0,4 & 0,6 & 0,6 \\
\hline$\left(x_{4}, x_{5}\right)$ & 0,7 & 0,5 & 0,7 & 0,4 \\
\hline
\end{tabular}

According to the algorithm construct fuzzy time-expanded static graph due to the step 1 and the rule 1 of the proposed method, as shown in the Fig. 3.

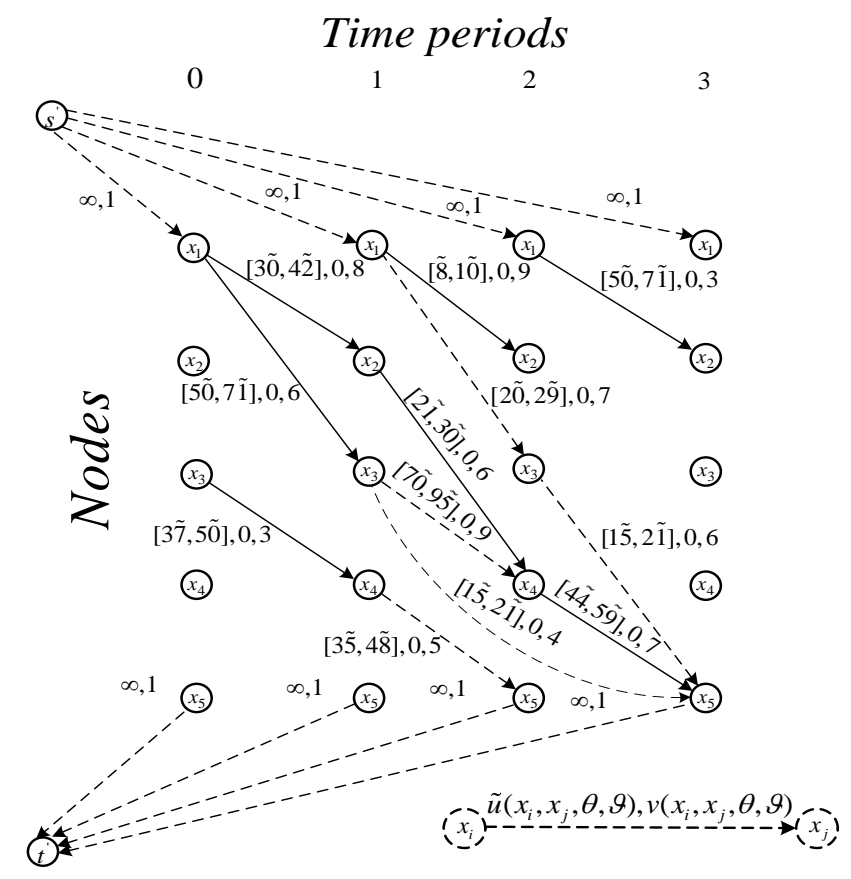

Fig. 3. $\tilde{G}_{p}-\ll$ time-expanded» graph $\tilde{G}$

Consequently finding the paths and passing the flow among them according to the steps of the proposed algorithm, obtain a time-expanded graph with the maximum flow in the Fig. 4. 


\section{Time periods}

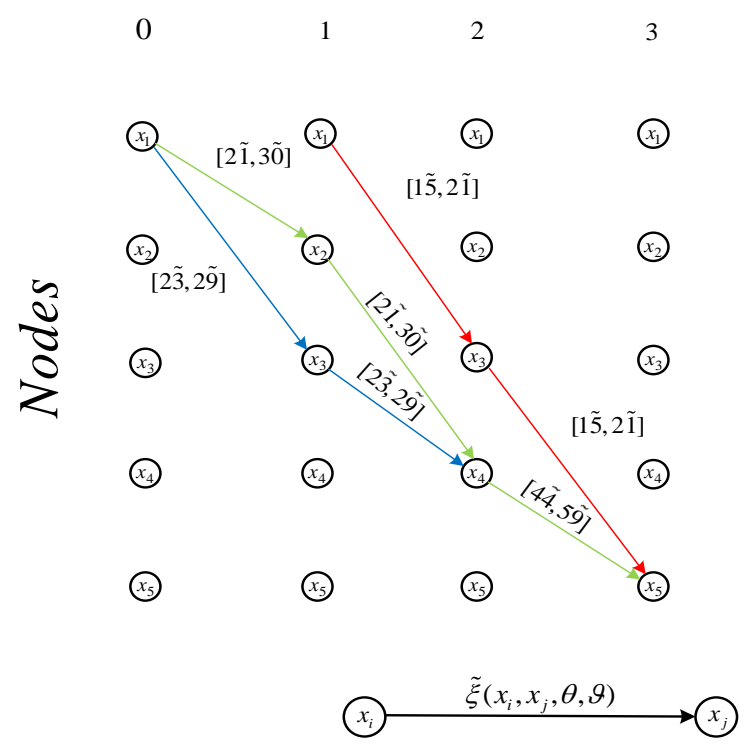

Fig. 4. $\tilde{G}_{p}$ with the maximum flow and vitality degree no less than 0,6

Finally, the maximum flow with the vitality degree no less than 0,6 that equals $[1 \tilde{5}, 2 \tilde{1}]+[2 \tilde{1}, 3 \tilde{0}]+[2 \tilde{3}, 2 \tilde{9}]=[5 \tilde{9}, 8 \tilde{0}]$ flow units in the time-expanded graph $\tilde{G}_{p}$ is obtained.

Let us define deviation borders of the obtained fuzzy interval $[5 \tilde{9}, 8 \tilde{0}]$ corresponded to the maximum flow in the graph $\tilde{G}$ according to the basic values of arc capacities in Fig. 5.

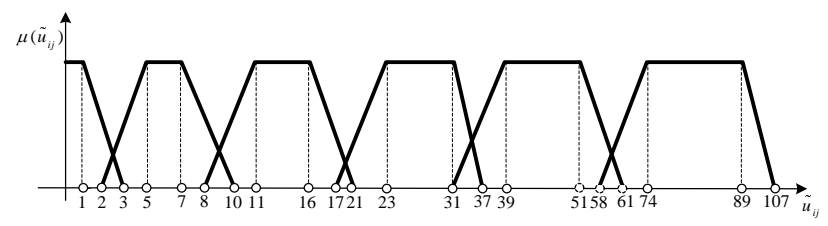

Fig. 5. Membership functions of the basic values of arc capacities of the network $\tilde{G}$

The detected result is between two adjacent basic values of the arc capacities: $[3 \tilde{9}, 5 \tilde{1}]$ with the left deviation $l_{1}^{L}=8$, the right deviation $-l_{1}^{R}=10$ and $[7 \tilde{4}, 89 \tilde{9}]$ with the left deviation $l_{2}^{L}=16$, the right deviation $-l_{2}^{R}=18$. According to (7)-(8) we obtain: $l_{1}^{L} \approx 12, l_{1}^{R} \approx 16$.

Therefore, the maximum flow in the fuzzy network with the vitality degree no less than 0,6 is obtained, which can be represented as a fuzzy trapezoidal number of $(59,80,12,16)$ units.

\section{CONCLUSION AND FUTURE WORK}

The task of the maximum flow finding in the fuzzy dynamic network with the given vitality degree is considered in the paper. The network's parameters are transit that allows us to call the described network as dynamic. Vitality parameters assigned to the arcs of the network are either of transit character. The formal method, based on the proposed definitions and rules is introduced. The solution of the problem has important practical value and uses the search of the maximum amount of cargo transportation taking into account different capacities of the roads at distinct periods of time and reliability of the system, expressed as vitality degree. In the future works we are going to propose methods of finding the maximum and the minimum cost flow in the fuzzy dynamic networks with the given vitality degree and nonzero lower flow bounds, methods of increasing the vitality degree in fuzzy dynamic networks.

\section{REFERENCES}

[1] A. Bozhenyuk, E. Gerasimenko, I. Rozenberg, and I. Perfilieva, "Method for the minimum cost maximum flow determining in fuzzy dynamic network with nonzero flow bounds," in proceedings of the 2015 Conference of the International Fuzzy Systems Association and the European Society for Fuzzy Logic and Technology, $30^{\text {th }}$ June- $3^{\text {rd }}$ July, Gijón, Asturias (Spain), vol. 89, José M. Alonso, H. Bustince, M. Reformat, Eds: Atlantic Press, 2015, pp. 385-392.

[2] H. Frank and I. T Frisch,.Communication, Transmission, and Transportation Networks: Addison Wesley, 1971.

[3] L.R. Ford and D.R. Fulkerson, Flows in Networks. Princeton: Princeton University Press, 1962.

[4] M. R. Silver and O. L. de Weck, "Time-expanded decision networks: A framework for designing evolvable complex systems," Systems Engineering, vol. 10, no. 2, 2007, pp. 167-188.

[5] E. Minieka, Optimization Algorithms for Networks and Graphs. New York and Basel: Marcel Dekker, Inc, 1978.

[6] J.E. Aronson, "A survey of dynamic network flows," Annals of Operations Research, vol. 20, 1989, pp. 1-66.

[7] J.B. Orlin, "Maximum-throughput dynamic network flows," Mathematical Programming. North-Holland, vol. 27, 1983. pp. 214-231.

[8] A. Bozhenyuk and E. Gerasimenko, "Flows finding in networks in fuzzy conditions," in Supply Chain Management Under Fuzzines, Studies in Fuzziness and Soft Computing, vol. 313, part III, Cengiz Kahraman and Basar Öztaysi, Eds. Berlin, Heidelberg: Springer-Verlag, 2014, pp. 269 291.

[9] A. Bozhenyuk, E. Gerasimenko, and I. Rozenberg, "Determining the minimum cost flow in fuzzy dynamic network with GIS «ObjectLand»," in Proceedings of the $20159^{\text {th }}$ International Conference on Application of Information and Communication Technologies (AICT), 14-16 October. Rostov-on-Don, Russia, 2015, pp. 294-298.

[10] A. Bozhenyuk and I. Rozenberg, "Allocation of Service Centers in the GIS with the Largest Vitality Degree," Advances in Computational Intelligence IPMU 2012, Part II, Series: Communications in Computer and Information Science, vol. 298, S. Greco et al., Eds. Berlin Heidelberg: Springer-Verlag, 2012, pp. 98-106.

[11] V. Bozheniuk, A. Bozhenyuk, and S. Belyakov, "Optimum allocation of centers in fuzzy transportation networks with the largest vitality degree," in: Proceedings of the 2015 Conference of the International Fuzzy System Association and the European Society for Fuzzy Logic and Technology: Atlantis Press, 2015, pp. 1006-1011.

[12] E. Miller-Hooks and S.S. Patterson, "On Solving quickest time problems in time-dependent dynamic networks," Journal of Mathematical Modeling and Algorithms, vol. 3, 2004, pp. 39-71. 
